\title{
Growth of Semimetals Bismuth and Antimony Films on Reactive Substrate
}

\author{
Xiao Wang, ${ }^{1}$ Xiaoxing Wei, ${ }^{2}$ and Caixia Song ${ }^{2}$ \\ ${ }^{1}$ State Key Laboratory of Marine Coatings, Marine Chemical Research Institute Co. Ltd., Qingdao 266071, China \\ ${ }^{2}$ College of Chemistry and Molecular Engineering, Qingdao University of Science \& Technology, Qingdao 266042, China
}

Correspondence should be addressed to Caixia Song; songcaixia@qingdaonews.com

Received 7 July 2014; Accepted 23 August 2014

Academic Editor: Yuanlie Yu

Copyright (C) 2015 Xiao Wang et al. This is an open access article distributed under the Creative Commons Attribution License, which permits unrestricted use, distribution, and reproduction in any medium, provided the original work is properly cited.

Semimetal Bi and Sb thin films with novel hierarchical structures were synthesized on zinc substrate via a hydrothermal method. $\mathrm{X}$-ray diffraction (XRD) analysis confirmed the formation of pure semimetals Bi and Sb. Scanning electron microscopy images showed that Bi films constructed with microtube arrays and hierarchical microspheres can be obtained selectively by altering the concentration of $\mathrm{Bi}^{3+}$ ions. The synthesized $\mathrm{Sb}$ films were constructed with bowl-shaped particles. The growth process of these semimetal architectures was briefly discussed.

\section{Introduction}

In recent years, shape controlled synthesis of metal materials in the nano-/microdimensions has been one of the important topics because these materials generally exhibit many size-, shape-, and structure-dependent properties. In particular, semimetal bismuth has attracted great attention because of its low carrier density, small effective mass, and large mean-free path, making it an interesting system for studying quantum confinement effects $[1,2]$. In addition, nanostructured bismuth could exhibit even more interesting electronic and thermoelectric properties $[3,4]$. Stimulated by these interesting properties, a number of different methods have been developed to prepare various bismuth nanostructures. Single crystal filaments of bismuth with diameters in the micron range have been prepared by Glocker and Skove [5]. Bi nanowires and nanorods were prepared by high pressure injection of the liquid metal or vapor phase of bismuth into the channels of porous anodic alumina template $[6,7]$. Bismuth nanowires have also been electrodeposited into nanometer-sized pores of polymer [8] or nanoporous anodic alumina $[9,10]$. Solution chemical methods have also been explored for the synthesis of Bi nanowires and nanotubes [11-13]. Wang et al. reported the synthesis of $\mathrm{Bi}$ nanoplates, nanorods, and nanoribbons from the precursor $\mathrm{Bi}\left[\mathrm{N}\left(\mathrm{SiMe}_{3}\right)_{2}\right]_{3}[14]$. Bismuth nanoparticles have been prepared in reverse micelles [15], by thermolysis [16], and through solution reduction [17-19]. Schulz et al. prepared Bi pseudocubes by thermal decomposition of a metal organic precursor [20]. The crystal structure of semimetal $\mathrm{Sb}$ is similar to that of $\mathrm{Bi}$, but the effective mass components of the electron ellipsoids in $\mathrm{Sb}$ are much larger than those in Bi. Clear optical switching of Sb thin films was observed under microscopic measurement [21]. Sb nanowires have been prepared similarly by vapor deposition [22], pulsed electrodeposition techniques [23], and solvothermal method [24]. Recently, Liu et al. prepared 3D nanostructured antimony in $\mathrm{SbCl}_{3}-(\mathrm{n}-\mathrm{Bu})_{4} \mathrm{NBF}_{4}-\mathrm{DMSO}$ solution [25].

Although several reports have been concerned with solution chemistry synthesis of semimetal bismuth and antimony nanocrystals, the synthesis of Bi tubular arrays and flower-shaped crystals and Sb nanobowls, to the best of our knowledge, has not been reported in the literature. Previously, we reported the synthesis of $\mathrm{ZnO}$ nanorod arrays on zinc substrate via hydrothermal method [26]. Herein we extend this hydrothermal method to the synthesis of semimetal bismuth and antimony films with hierarchical structures. 


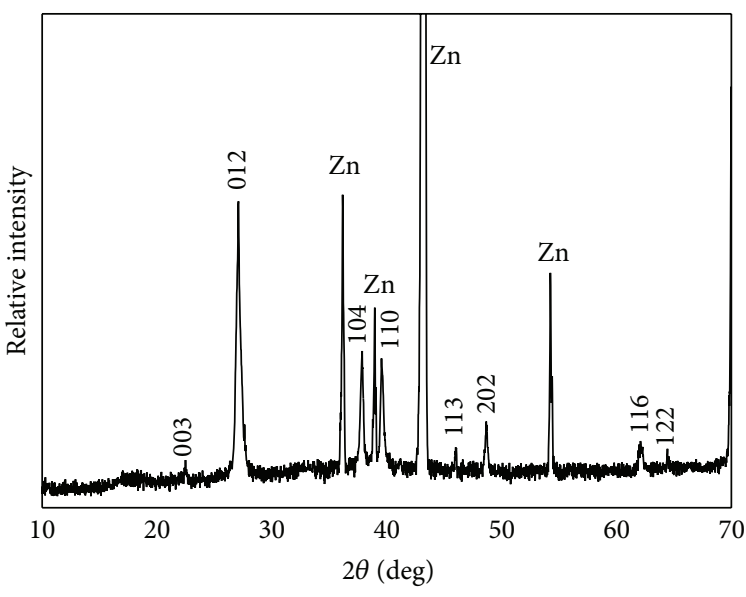

(a)

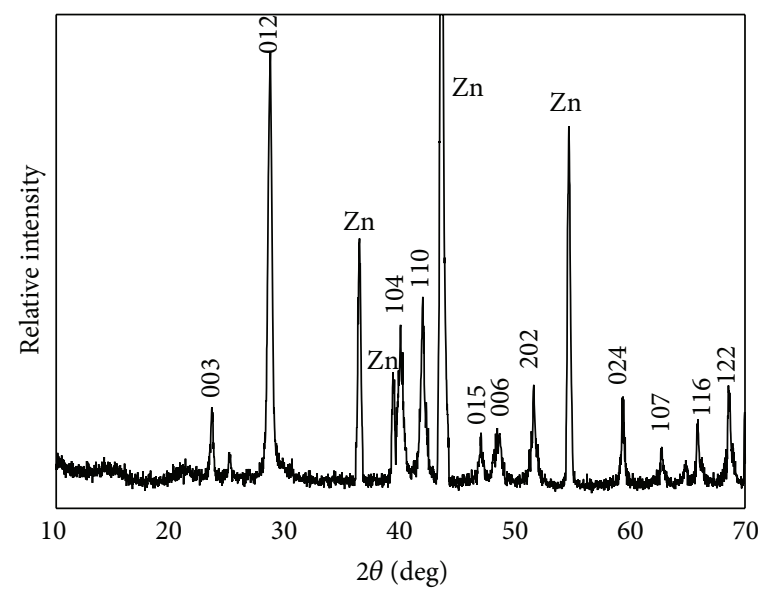

(b)

Figure 1: XRD patterns of $\mathrm{Bi}\left((\mathrm{a}) 120^{\circ} \mathrm{C}, 10 \mathrm{~h}, 4.6 \mathrm{mmol} / \mathrm{L}\right.$ of $\left.\mathrm{Bi}^{3+}\right)$ and $\mathrm{Sb}\left((\mathrm{b}) 150^{\circ} \mathrm{C}, 24 \mathrm{~h}, 2.8 \mathrm{mmol} / \mathrm{L}\right.$ of $\left.\mathrm{Sb}^{3+}\right)$.

\section{Experimental Materials and Methods}

Fresh zinc foils $(15 \mathrm{~mm} \times 15 \mathrm{~mm})$ with a purity of $99.9 \%$ were used as both a reagent and a substrate for the direct growth of bismuth microtube arrays. In a typical process, analytically pure $\mathrm{BiCl}_{3}$ and poly-(vinylpyrrolidone) (PVP, M.W. 10000) were dispersed in deionized water under vigorous stirring; then the $\mathrm{pH}$ value was adjusted to 7.0 by addition of diluted aqueous $\mathrm{NaOH}$. The mixture was transferred into a Teflonlined stainless steel autoclave up to $85 \%$ of its capacity. The zinc foils were carefully washed with absolute alcohol and deionized water by ultrasound. After the zinc substrate was dipped into the solution, the autoclave was sealed and put into an oven. The reaction was conducted at $120^{\circ} \mathrm{C}$ for $10 \mathrm{~h}$. After that, the zinc foils were taken out and rinsed with deionized water and alcohol. Bowl-shaped antimony nanostructures were prepared in the same way, just replacing $\mathrm{BiCl}_{3}$ with $\mathrm{SbCl}_{3}$.

The as-prepared products on the substrate were directly subjected to characterizations by scanning electron microscopy (SEM) and powder X-ray diffraction (XRD). The XRD patterns were recorded by employing a Philips X'pert X-ray diffractometer with $\mathrm{Cu}$ K-Alpha radiation $(\lambda=$ $0.154187 \mathrm{~nm}$ ). SEM images were obtained on a JSM-6700F field emission scanning electron microscope.

\section{Results and Discussion}

Figure 1 shows XRD pattern of the as-prepared bismuth and antimony samples. As shown in Figure 1(a), all the major peaks are labeled and can be attributed to rhombcentered bismuth metal according to the standard JCPDS diffraction file (cards number 5-519), except those marked with Zn coming from the zinc substrate. The calculated lattice parameters ( $a=0.454 \mathrm{~nm}$ and $c=1.184 \mathrm{~nm}$ ) are consistent with those reported in the JCPDS diffraction file, while XRD pattern in Figure 1(b) confirms the formation of rhombcentered metal antimony (JCPDS cards number 35-732), accompanied by those strong peaks marked with $\mathrm{Zn}$ coming from the zinc substrate.

The morphology of the as-prepared bismuth sample was examined by SEM. Figure 2(a) shows SEM image of the zinc foil. It can be seen that zinc foil has a relatively smooth surface. After being treated in $4.6 \mathrm{mmol} / \mathrm{L}$ of $\mathrm{Bi}^{3+}$, the entire surface of the zinc substrate is covered by a film of rod-like structures based on the SEM observation in Figure 2(b). It can be clearly seen from SEM image that only rod-like features are observed for the sample, which are quasi-aligned in a dense array. A higher magnification SEM image of bismuth rod arrays is shown in Figure 2(c). It can be clearly seen that the individual bismuth rod has a diameter in range of 300 $1000 \mathrm{~nm}$ and length of more than $10 \mu \mathrm{m}$ growing radially on zinc substrate. Some open-ended tubes are also observed in Figure 2(c). Close examination of the top tips of the bismuth rods reveals that most of the rod-like structures are in fact bismuth tubes (Figure 2(d)). The average diameter of the open-ended tubes is $\sim 600 \mathrm{~nm}$, with hollow centers typically measured to be $\sim 200 \mathrm{~nm}$ in diameter. Careful observation reveals that the walls of the tubes are constructed with large amount of flexible sheet-like tiny species in nanoscale.

The influence of the concentration of $\mathrm{Bi}^{3+}$ on the structures of the films has been investigated. When the synthesis was carried out in higher concentration of $\mathrm{Bi}^{3+}(5.8 \mathrm{mmol} / \mathrm{L})$, a large amount of spherical bismuth microstructures, instead of Bi tubular crystals, was produced (Figure 3(a)). Although most of the microspheres appear to be separate particles, some of them are partially fused together to form larger microsphere aggregates. The exterior surfaces of the microspheres are not smooth but contain extensively growing sheet-like structures. To further examine the surface structure of these microspheres, a high magnification SEM image was obtained and is shown in the inset of Figure 3(a). The microspheres are randomly grown from seemingly flexible nanosheets that can be bent and connected with each other. The thickness of these nanosheets mostly ranges between 80 and $120 \mathrm{~nm}$, whereas the size of the spherical structure is around $3 \mu \mathrm{m}$. When the concentration of $\mathrm{Bi}^{3+}$ was further 


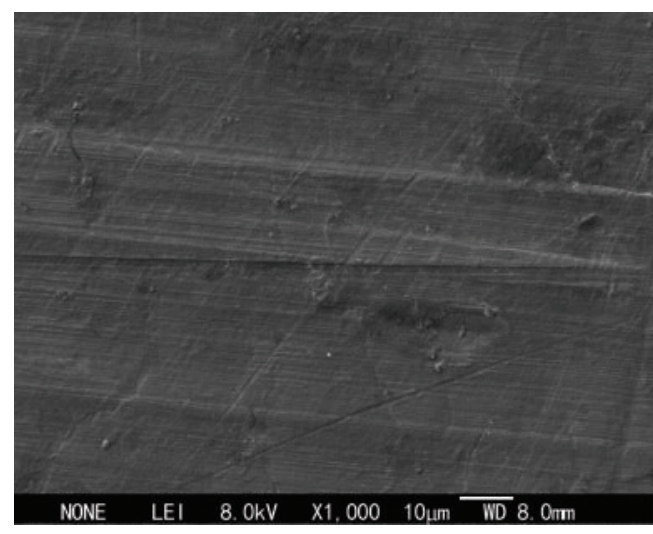

(a)

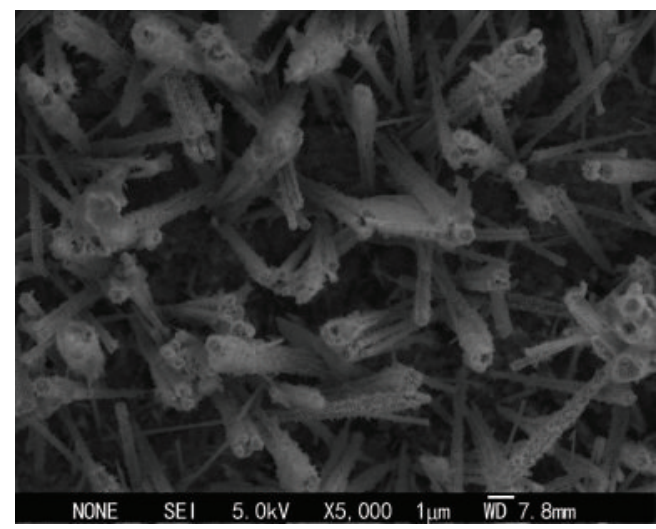

(c)

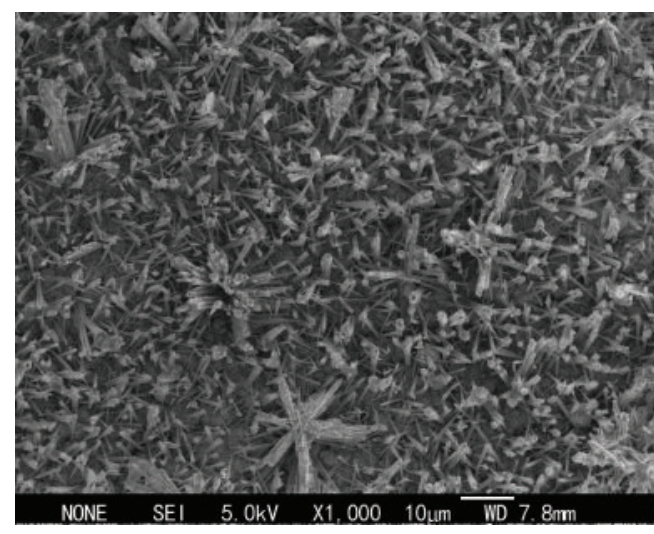

(b)

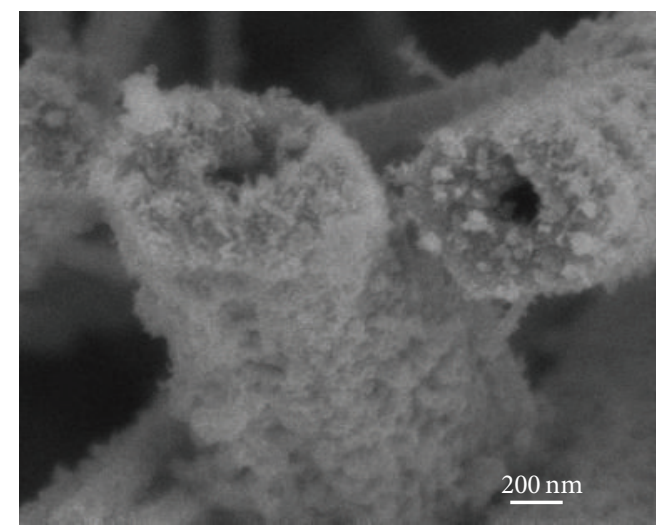

(d)

FIGURE 2: SEM images of zinc substrate (a) and Bi microtube arrays ((b)-(d) $120^{\circ} \mathrm{C}, 10 \mathrm{~h}, 4.6 \mathrm{mmol} / \mathrm{L}^{\circ} \mathrm{Bi}^{3+}$ ).

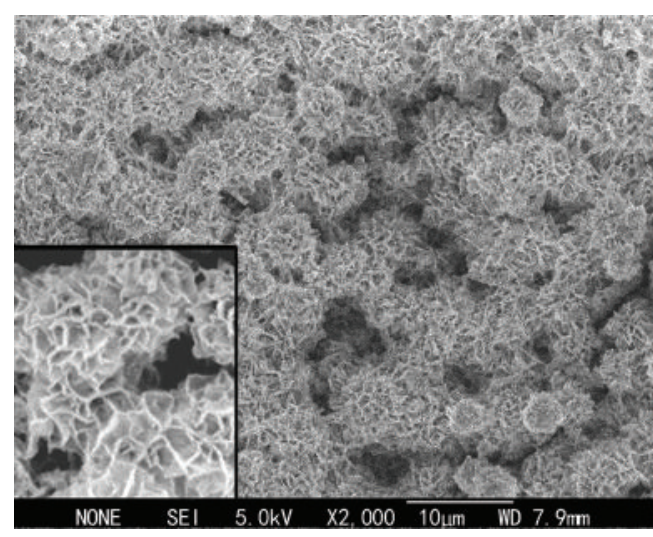

(a)

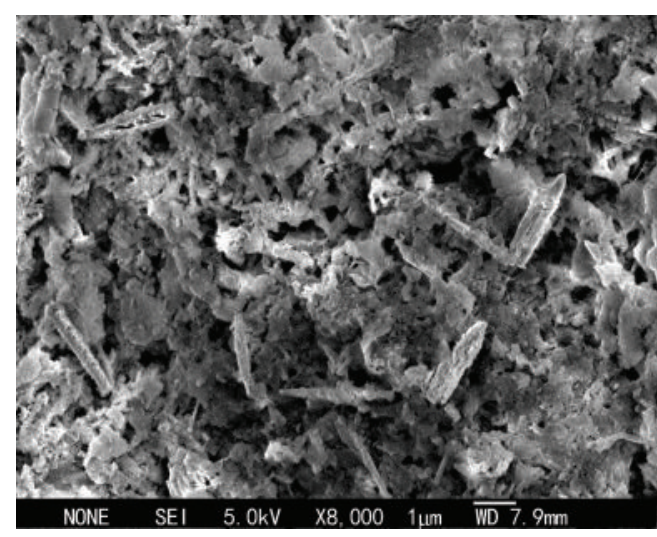

(b)

FIGURE 3: SEM images of Bi (a) flower-shaped crystal and (b) sheet-like structures. Inset of (a) shows magnified image of the spheres.

increased to $7.0 \mathrm{mmol} / \mathrm{L}$, SEM image shown in Figure 3(b) revealed that the film was constructed with randomly stacked sheet-like structures with irregular shapes.

The formation of bismuth nanotubes in aqueous hydrazine system has been thought to be the result of the rolling of its pseudolayered structure [11]. As for the growth of tubular structures on substrate, the nuclei patterns on the substrates were deduced to be the mechanism for the formation of tube architectures. It has been reported that ring-like patterned nuclei agglomeration results in the growth of tubular structures [27]. In the present study, the hydrothermal reaction of bismuth crystals can be explained as follows:

$$
\begin{gathered}
\mathrm{M}^{3+}+\mathrm{Cl}^{-}+\mathrm{H}_{2} \mathrm{O} \rightleftharpoons \mathrm{MOCl}+2 \mathrm{H}^{+} \\
\mathrm{M}^{3+}+3 \mathrm{OH}^{-} \rightleftharpoons \mathrm{M}(\mathrm{OH})_{3} \\
2 \mathrm{M}^{3+}+3 \mathrm{Zn} \longrightarrow 2 \mathrm{M}+3 \mathrm{Zn}^{2+}
\end{gathered}
$$

$(\mathrm{M}=\mathrm{Bi}, \mathrm{Sb})$. 


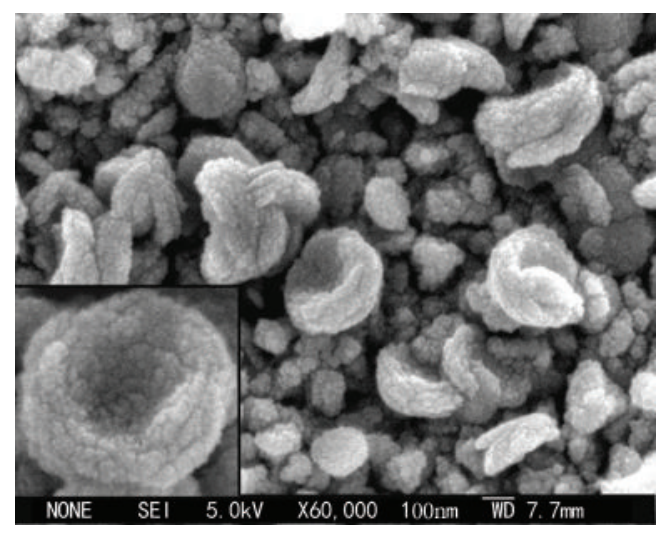

(a)

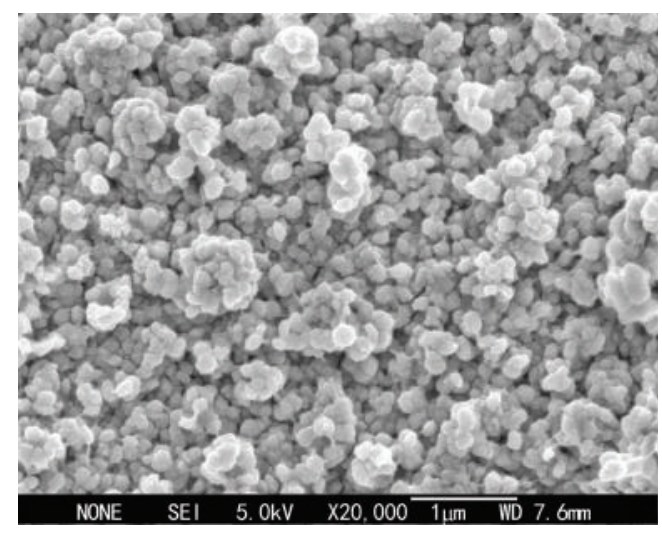

(b)

FIGURE 4: SEM images of $\mathrm{Sb}$ films prepared at $150^{\circ} \mathrm{C}$ for $24 \mathrm{~h}$ with (a) 2.8 and (b) $4.8 \mathrm{mmol} / \mathrm{L}$ of Sb ${ }^{3+}$. Inset of (a): a magnified image of one bowl.

$\mathrm{Bi}^{3+}$ ions have higher reaction activity with metal $\mathrm{Zn}$; thus it is necessary to reduce the concentration of $\mathrm{Bi}^{3+}$ ions by adjusting the $\mathrm{pH}$ values. And then $\mathrm{BiCl}_{3}$ hydrolyzed into $\mathrm{BiOCl}$ and/or $\mathrm{Bi}(\mathrm{OH})_{3}$. Controlled release of $\mathrm{Bi}^{3+}$ ions in reactions (1) and (2) was suitable for the reduction of $\mathrm{Bi}^{3+}$ into $\mathrm{Bi}$ nuclei. And the in situ formed Bi nuclei or nanoparticles may serve as seeds for the continuous epitaxial growth of Bi nuclei into tubular structures on the surface of zinc foil, which is similar to those tubular structures resulting from ring-like patterned nuclei agglomeration [27]. Along with the growth of Bi tubes, secondary nucleation may take place on their surface, which leads to the branched growth of sheet-like species on the tubes surface and thickening the walls of the tubes, whereas at higher concentration of $\mathrm{Bi}^{3+}$ ions with higher reaction velocity, the nucleation and crystal growth occur at a faster rate. As a result, nanosheets related Bi nanostructures were formed which might be contributed to the pseudolayered nature of metal bismuth.

It is known that antimony has a pseudolayered arsenic structure similar to that of rhombohedral bismuth. By extending this method to the preparation of antimony, however, bowl-shaped aggregates of $\mathrm{Sb}$ nanoparticles were obtained $\left(150^{\circ} \mathrm{C}, 2.8 \mathrm{mmol} / \mathrm{L} \mathrm{Sb}^{3+}\right)$. Figure $4(\mathrm{a})$ shows the SEM image of the antimony film. It clearly reveals that zinc substrate was covered with Sb nano- or microparticles. Most of the particles exhibit bowl-shaped morphologies and display size in the range of 100-500 nm and wall thickness of about $80 \mathrm{~nm}$. Higher magnification SEM image of an individual $\mathrm{Sb}$ nanobowl in the inset of Figure 4(a) reveals that the $\mathrm{Sb}$ nanobowl was constructed with even smaller Sb nanoparticles of $10-20 \mathrm{~nm}$ in diameters. Control experimental results show that the concentrations of $\mathrm{Sb}^{3+}$ have much influence on the structure of the films. If the reaction was conducted at higher concentration of $\mathrm{Sb}^{3+}(4.8 \mathrm{mmol} / \mathrm{L})$, SEM image in Figure 4(b) reveals that the thin film is also constructed with nanoparticles and their aggregates, exhibiting irregular spherical shapes and wide size distributions.

More experimental results showed that the asprepared products were sensitive to the initial $\mathrm{pH}$ value of the suspension. If initial $\mathrm{pH}$ value was higher than 8 , $\mathrm{ZnO}$ can be detected in the samples, while at lower initial $\mathrm{pH}$ values, such as a value lower than 6.0, the reaction activity between zinc and $\mathrm{Bi}^{3+}\left(\mathrm{Sb}^{3+}\right)$ ions was higher; as a result, irregularly aggregated nanostructures were obtained. When the hydrothermal reaction was conducted with initial $\mathrm{pH}$ value of 7.0, desired samples were obtained and the $\mathrm{pH}$ value slightly decreased to the range of 5.0-6.0. Additional experiments aimed at elucidating different growth mechanisms for metal $\mathrm{Bi}$ and $\mathrm{Sb}$ are in progress in our laboratory.

\section{Conclusion}

In summary, a convenient hydrothermal route has been developed to prepare microtube arrays and hierarchical microspheres of metal Bi and bowl-shaped particles of metal $\mathrm{Sb}$. The morphologies of $\mathrm{Bi}$ and $\mathrm{Sb}$ metal crystalline films were mainly controlled by the concentrations of metal ions and the initial $\mathrm{pH}$ values of the suspension. Extensive studies on different growth mechanisms for metals $\mathrm{Bi}$ and $\mathrm{Sb}$ are needed. The preparation strategy presented here is facile and cost-effective, which may open new avenues for the design of functional thin films of metal or other inorganic materials.

\section{Conflict of Interests}

The authors declare that there is no conflict of interests regarding the publication of this paper.

\section{Acknowledgments}

This work was financially supported by the Natural Science Foundation of Shandong Province (ZR2011EMM007, ZR2013BM002) and the Natural Science Found of Qingdao (12-1-4-3-(13)-jch). 


\section{References}

[1] T. E. Huber, M. J. Graf, C. A. Foss Jr., and P. Constant, "Processing and characterization of high-conductance bismuth wire array composites," Journal of Materials Research, vol. 15, no. 8, pp. 1816-1821, 2000.

[2] Y. W. Wang, J. S. Kim, G. H. Kim, and K. S. Kim, "Quantum size effects in the volume plasmon excitation of bismuth nanoparticles investigated by electron energy loss spectroscopy," Applied Physics Letters, vol. 88, no. 14, Article ID 143106, 2006.

[3] E. Condrea and A. Nicorici, "Quantum size effect in the resistivity of bismuth nanowires," Solid State Communications, vol. 150, no. 1-2, pp. 118-121, 2010.

[4] H. Zhang, J. S. Son, J. Jang et al., " $\mathrm{Bi}_{1-x} \mathrm{Sb}_{x}$ alloy nanocrystals: colloidal synthesis, charge transport, and thermoelectric properties," ACS Nano, vol. 7, no. 11, pp. 10296-10306, 2013.

[5] D. A. Glocker and M. J. Skove, "Field effect and magnetoresistance in small bismuth wires," Physical Review B, vol. 15, no. 2, pp. 608-616, 1977.

[6] Z. Zhang, D. Gekhtman, M. S. Dresselhaus, and J. Y. Ying, "Processing and characterization of single-crystalline ultrafine bismuth nanowires," Chemistry of Materials, vol. 11, no. 7, pp. 1659-1665, 1999.

[7] J. Reppert, R. Rao, M. Skove et al., "Laser-assisted synthesis and optical properties of bismuth nanorods," Chemical Physics Letters, vol. 442, no. 4-6, pp. 334-338, 2007.

[8] K. Liu, C. L. Chien, and P. C. Searson, "Finite-size effects in bismuth nanowires," Physical Review B: Condensed Matter and Materials Physics, vol. 58, no. 22, Article ID R14681, 1998.

[9] Y. Peng, D. H. Qin, R. J. Zhou, and H. L. Li, "Bismuth quantumwires arrays fabricated by electrodeposition in nanoporous anodic aluminum oxide and its structural properties," Materials Science and Engineering B: Solid-State Materials for Advanced Technology, vol. 77, no. 3, pp. 246-249, 2000.

[10] Y. Zhu, X. Dou, X. Huang, A. Li, and G. Li, "Thermal properties of Bi nanowire arrays with different orientations and diameters," The Journal of Physical Chemistry B, vol. 110, no. 51, pp. 2618926193, 2006.

[11] Y. Li, J. Wang, Z. Deng et al., "Bismuth nanotubes: A rational low-temperature synthetic route," Journal of the American Chemical Society, vol. 123, no. 40, pp. 9904-9905, 2001.

[12] J. Wang, X. Wang, Q. Peng, and Y. Li, "Synthesis and characterization of bismuth single-crystalline nanowires and nanospheres," Inorganic Chemistry, vol. 43, no. 23, pp. 75527556, 2004

[13] B. Yang, C. Li, H. Hu, X. Yang, Q. Li, and Y. Qian, "A roomtemperature route to bismuth nanotube arrays," European Journal of Inorganic Chemistry, vol. 2003, no. 20, pp. 3699-3702, 2003.

[14] F. Wang, R. Tang, H. Yu, P. C. Gibbons, and W. E. Buhro, "Size- and shape-controlled synthesis of bismuth nanoparticles," Chemistry of Materials, vol. 20, no. 11, pp. 3656-3662, 2008.

[15] E. E. Foos, R. M. Stroud, A. D. Berry, A. W. Snow, and J. P. Armistead, "Synthesis of nanocrystalline bismuth in reverse micelles," Journal of the American Chemical Society, vol. 122, no. 29, pp. 7114-7115, 2000.

[16] Z. Wang, C. Jiang, R. Huang, H. Peng, and X. Tang, "Investigation of optical and photocatalytic properties of bismuth nanospheres prepared by a facile thermolysis method," The Journal of Physical Chemistry C, vol. 118, no. 2, pp. 1155-1160, 2014.
[17] D. Ma, J. Zhao, R. Chu et al., "Novel synthesis and characterization of bismuth nano/microcrystals with sodium hypophosphite as reductant," Advanced Powder Technology, vol. 24, no. 1, pp. 79-85, 2013.

[18] W. Z. Wang, B. Poudel, Y. Ma, and Z. F. Ren, "Shape control of single crystalline bismuth nanostructures," The Journal of Physical Chemistry B, vol. 110, no. 51, pp. 25702-25706, 2006.

[19] Y. W. Wang, B. H. Hong, and K. S. Kim, "Size control of semimetal bismuth nanoparticles and the UV - Visible and ER absorption spectra," Journal of Physical Chemistry B, vol. 109, no. 15, pp. 7067-7072, 2005.

[20] S. Schulz, S. Heimann, C. Wölper, and W. Assenmacher, "Synthesis of bismuth pseudocubes by thermal decomposition of $\mathrm{Bi}_{2} \mathrm{Et}_{4}$," Chemistry of Materials, vol. 24, no. 11, pp. 2032-2039, 2012.

[21] T. Fukaya, J. Tominaga, T. Nakano, and N. Atoda, "Optical switching property of a light-induced pinhole in antimony thin film," Applied Physics Letters, vol. 75, no. 20, pp. 3114-3116, 1999.

[22] J. Heremans, C. M. Thrush, Y.-M. Lin, S. B. Cronin, and M. S. Dresselhaus, "Transport properties of antimony nanowires," Physical Review B, vol. 63, no. 8, Article ID 085406, 2001.

[23] Y. Zhang, G. Li, Y. Wu, B. Zhang, W. Song, and L. Zhang, "Antimony nanowire arrays fabricated by pulsed electrodeposition in anodic alumina membranes," Advanced Materials, vol. 14, no. 17, pp. 1227-1230, 2002.

[24] Y. W. Wang, B. H. Hong, J. Y. Lee, J.-S. Kim, G. H. Kim, and K. S. Kim, "Antimony nanowires self-assembled from Sb nanoparticles," Journal of Physical Chemistry B, vol. 108, no. 43, pp. 1672316726, 2004.

[25] P. Liu, K. Zhong, C. Liang et al., "Self-assembly of threedimensional nanostructured antimony," Chemistry of Materials, vol. 20, no. 24, pp. 7532-7538, 2008.

[26] D. Wang and C. Song, "Controllable synthesis of $\mathrm{ZnO}$ nanorod and prism arrays in a large area," The Journal of Physical Chemistry B, vol. 109, no. 26, pp. 12697-12700, 2005.

[27] S. Sharma and M. K. Sunkara, "Direct synthesis of gallium oxide tubes, nanowires, and nanopaintbrushes," Journal of the American Chemical Society, vol. 124, no. 41, pp. 12288-12293, 2002. 

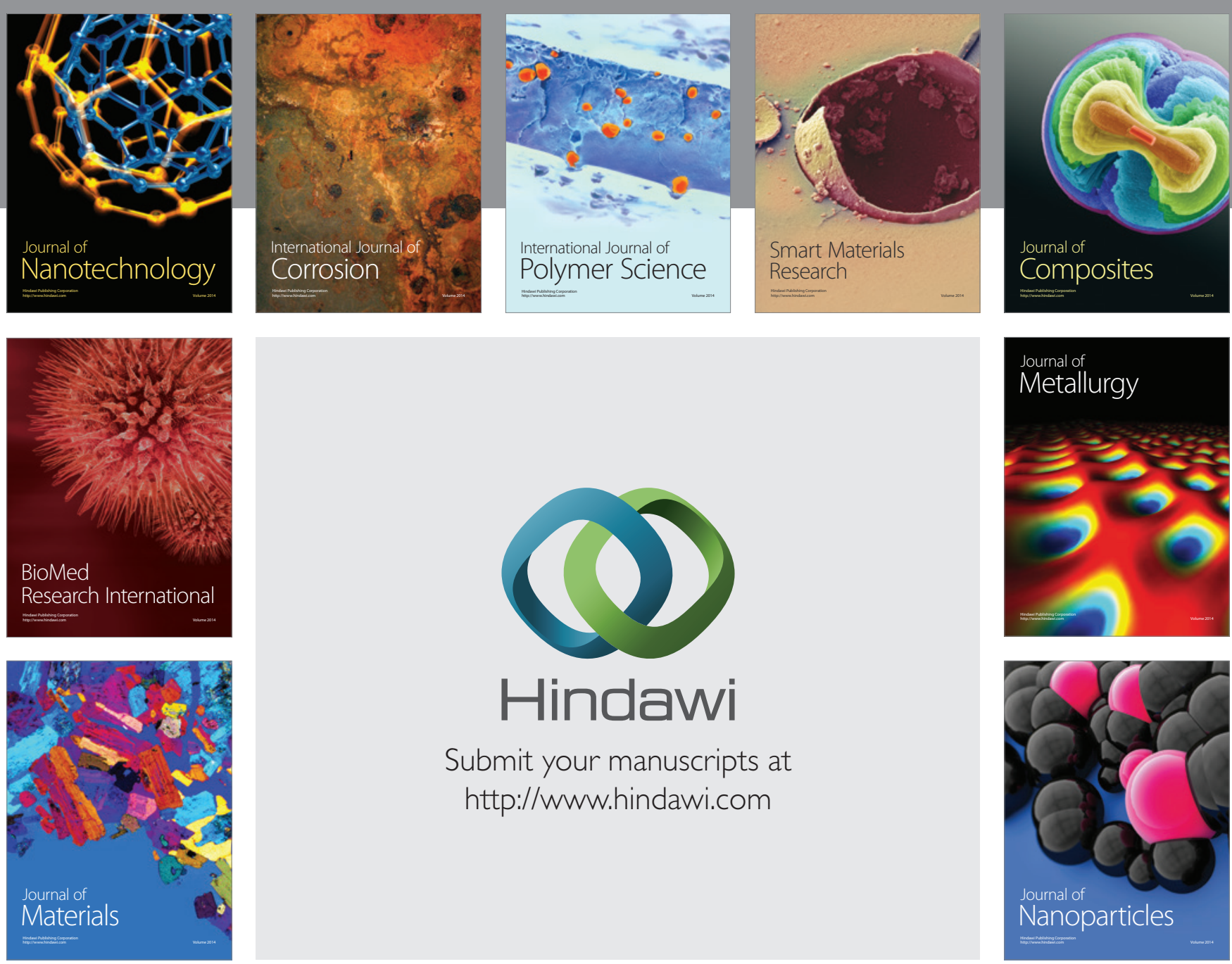

Submit your manuscripts at http://www.hindawi.com
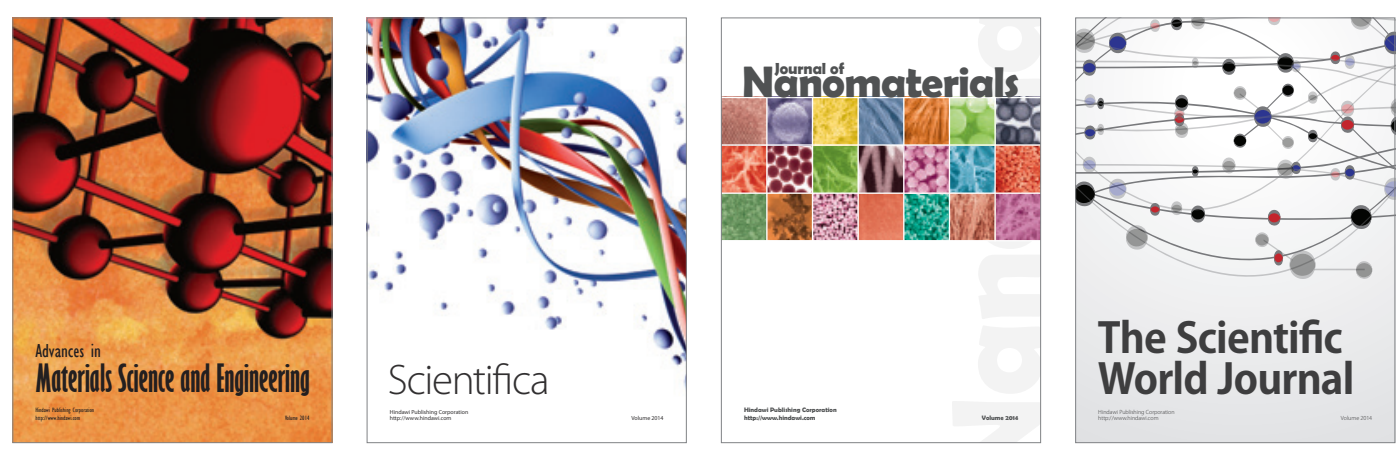

\section{The Scientific World Journal}
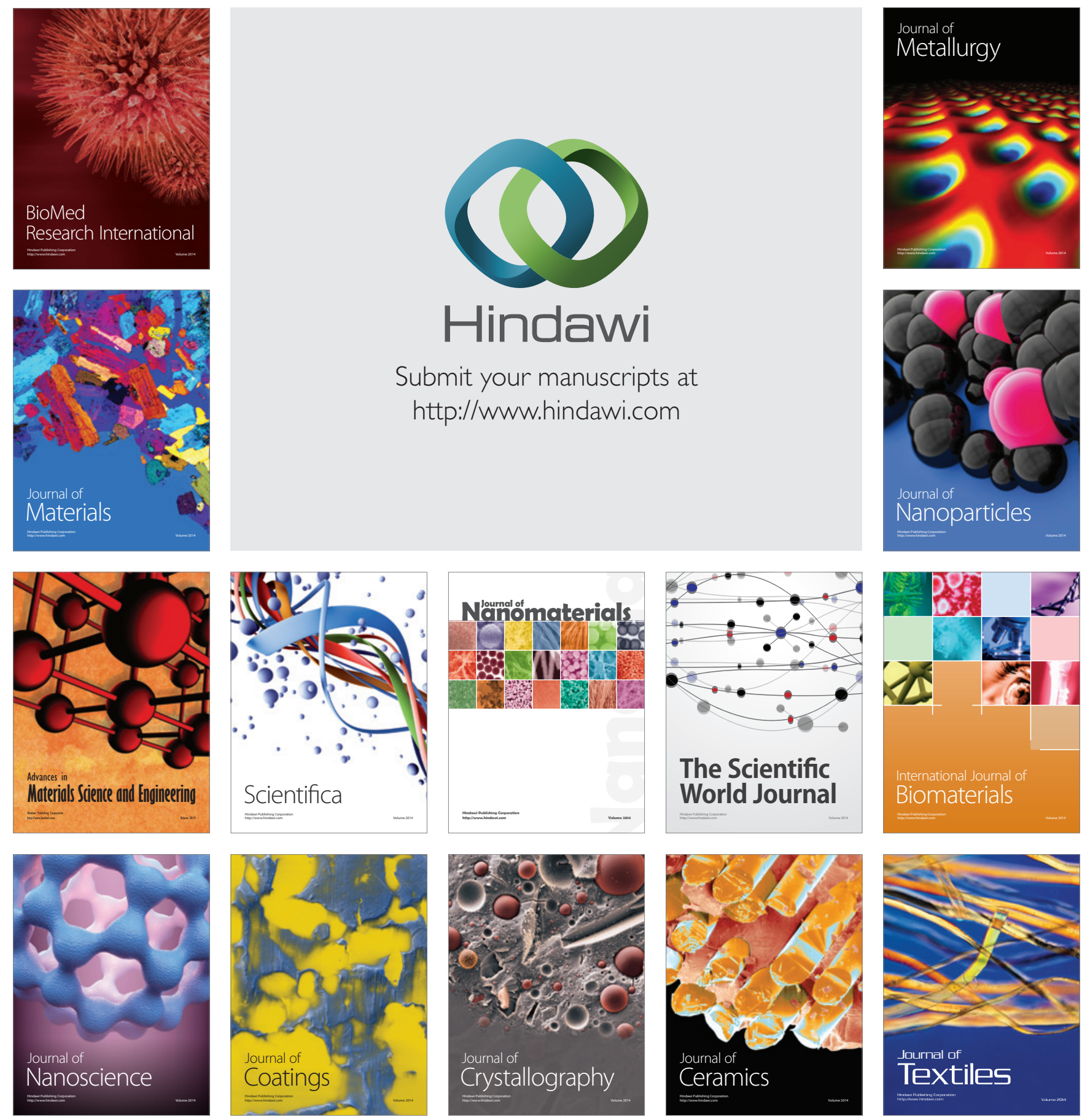